\title{
ION ENERGETICS IN THE INNER COMA OF COMET HALLEY
}

\author{
T. E. Cravens
}

Space Physics Research Laboratory and Department of Atmospheric and Oceanic

Science, The University of Michigan, Ann Arbor, MI 48109, U.S.A.

\begin{abstract}
The cometary plasma in the magnetic barrier just outside the diamagnetic cavity which surrounds the nucleus of comet Halley is virtually stagnant. The outflowing neutral gas exerts an outward ion-neutral drag force on this plasma, which balances the inward magnetic pressure gradient force in the vicinity of the contact surface. The cometary ions are frictionally heated due to the relative motion of the ion and neutral gases. The ion flow velocity must have a few $\mathrm{km} / \mathrm{s}$ non-radial component in order to explain the ion temperatures measured by the ion mass spectrometer on Giotto.
\end{abstract}

\section{Introduction}

The plasma in the inner coma of comet Halley is almost stationary, with flow speeds less than a few $\mathrm{km} / \mathrm{s}$, and is predominantly of cometary origin (Gringauz et al.; 1986; Balsiger et al., 1986). Well within the stagnation region, a field-free (diamagnetic) cavity was observed by the magnetometer onboard the Giotto spacecraft during its encounter with comet Halley (Neubauer et al., 1986). The boundary of this cavity (which has been called the contact surface, or CS) is located at a cometocentric distance of about $4000 \mathrm{~km}$. The ion velocities measured within the cavity by the ion mass spectrometer (IMS) onboard Giotto (Balsiger et al., 1986) are about the same as the neutral velocity $\left(u_{n}=0.93\right.$ $\mathrm{km} / \mathrm{s}$ )(Krankowsky et al., 1986), whereas just outside the CS, the measured ion velocities (actually just the components along the almost radial Giotto trajectory) are almost zero (see Figure 1).

The ion temperatures ( $\mathrm{T}_{\mathrm{i}}$ or just $\mathrm{T}$ ) measured by the IMS (Balsiger et al., 1986; Schwenn et al., 1986) or by the neutral mass spectrometer (NMS) (Lämmerzahl et al., 1986) are only $300-400 \mathrm{~K}$ inside the cavity, but jump up to $\approx 1500 \mathrm{~K}$ at the contact surface (Figure 1). Outside the CS, T increases with increasing cometocentric distance, $r$, and attains a value of approximately $2 \times 10^{4} \mathrm{~K}$ at $\mathrm{r}=3 \times 10^{4} \mathrm{~km}$. The total ion density measured by the IMS varies as $1 / \mathrm{r}$ for $\mathrm{r}$ less than $10^{4}$ $\mathrm{km}$ (Figure 1), and this indicates that the plasma density is photochemically controlled (cf., Mendis et al., 1985; Cravens, 1986a).

Haerendel (1987) recently suggested that significant ion heating occurs outside the $\mathrm{CS}$ due to the relative motion between the ions and neutrals, and he derived an analytic expression for T. In the present paper, I also examine the ion energy balance equation and derive an expression for the ion temperature. This expression includes an ion-neutral frictional heating term which is identical to that in Haerendel (1987) and which is well-known in the field of aeronomy (cf., Banks and Kockarts, 1973).

The present paper's treatment of cometary ion energetics differs from Haerendel's in two very important respects. First, the temperature expression used here is more accurate and includes a chemical heating term which is very important inside the diamagnetic cavity. Second, it will be suggested in this paper that the plasma motion in the inner coma of comet Halley had a significant non-radial component. The plasma velocities calculated by Haerendel $(1986,1987)$, on the other

Copyright 1987 by the American Geophysical Union.

Paper number 7L6594D

0094-8276/87/007L-6594\$03.00 hand, and used in his temperature expression, were assumed to be strictly radially inward. And his calculated radial velocities are much larger than the measured radial velocities (Figure 1). In this paper, I calculate the non-radial velocity semi-empirically using the measured radial velocities and the measured ion temperatures in conjunction with the theoretical temperature formula.

\section{Chemical Heating}

The neutral composition measured in the inner coma of comet Halley by the Giotto NMS (Krankowsky et al., 1986) was $80 \%$ or more $\mathrm{H}_{2} \mathrm{O}$ and a few $\% \mathrm{CO}_{2}$. The measured ion composition from both the IMS and NMS indicated that $\mathrm{H}_{3} \mathrm{O}^{+}$ was the major ion for $\mathrm{r}<2 \times 10^{4} \mathrm{~km}$, followed in abundance by $\mathrm{H}_{2} \mathrm{O}^{+}$and $\mathrm{OH}^{+}$. Obviously, water chemistry is dominant for comet Halley (Mendis et al., 1985; Cravens, 1986a; Körösmezey et al., 1987). $\mathrm{H}_{2} \mathrm{O}$ is photoionized by solar extreme ultraviolet radiation and can also be ionized by electron impact either by photoelectrons (giving a 10-15\% enhancement over photoionization) or by other electrons (cf., Cravens et al, 1987).

$$
\begin{array}{r}
\text { hv (or } \left.\mathrm{e}^{-}\right)+\mathrm{H}_{2} \mathrm{O}--->\mathrm{H}_{2} \mathrm{O}^{+}+\text {e } \quad(\approx 85 \%) \\
---->\mathrm{OH}^{+}+\mathrm{H}+\mathrm{e}(\approx 15 \%)
\end{array}
$$

The total ionization frequency for $\mathrm{H}_{2} \mathrm{O}$ at solar minimum is 4.5 $x 10^{-7} \mathrm{~s}^{-1}$. The $\mathrm{H}_{2} \mathrm{O}^{+}$and $\mathrm{OH}^{+}$ions react with $\mathrm{H}_{2} \mathrm{O}$ :

$$
\begin{aligned}
& \mathrm{H}_{2} \mathrm{O}^{+}+\mathrm{H}_{2} \mathrm{O}---->\mathrm{H}_{3} \mathrm{O}^{+}+\mathrm{OH}+\Delta \mathrm{E}_{1}
\end{aligned}
$$

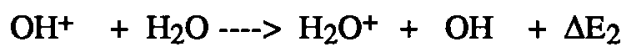

Values in the literature for the exothermic energy $\Delta E_{1}$ vary from $0.5 \mathrm{eV}$ (Banks and Kockarts, 1973) up to $1.9 \mathrm{eV}$ (Körösmezey et al., 1987); $1.0 \mathrm{eV}$ is used here. $\Delta \mathrm{E}_{2}=1.82$ eV (Mendis et al., 1985). $\mathrm{CO}_{2}^{+}$and $\mathrm{CO}^{+}$-associated chemistry also makes a modest contribution to the overall ion chemical heating and was taken into account.

The chemical ion heating rate (units of $\mathrm{ergs} / \mathrm{cm}^{3} / \mathrm{s}$ ) is:

$$
\mathrm{Q}_{\mathrm{ch}}=\mathrm{I}_{\mathrm{eff}} \Delta \mathrm{E}_{\mathrm{eff}} \mathrm{F} \mathrm{C}_{\mathrm{v}} \mathrm{n}_{\mathrm{n}}=\mathrm{k}_{\mathrm{Q}} \mathrm{n}_{\mathrm{n}}
$$

where $n_{n}$ is the neutral density. $I_{\text {eff }}=4.8 \times 10^{-7} \mathrm{~s}^{-1}$ is the average, or effective, ionization frequency. $\Delta \mathrm{E}_{\mathrm{eff}}=0.7 \mathrm{eV}$ is the energy given to the ion products for all relevant chemical reactions. $F$ is the fraction of the product ion energy that goes into heating other ions via Coulomb collisions rather than heating neutrals; $F$ is very close to unity in the inner coma. $\mathrm{C}_{\mathrm{V}}$ is the (unknown) fraction of the exothermic energy which appears as translational energy as opposed to internal (i.e., rotational and vibrational) excitation. Finally, $\mathrm{k}_{\mathrm{Q}}=6.1 \mathrm{x}$ $10^{-19} \mathrm{C}_{\mathrm{v}}$ ergs/s.

\section{Ion Energy Equation}

The ion energy equation relevant to a collisional, magnetized plasma can be found in the "conservation" form by taking the left-hand side of the Schmidt and Wegmann (1982) energy 

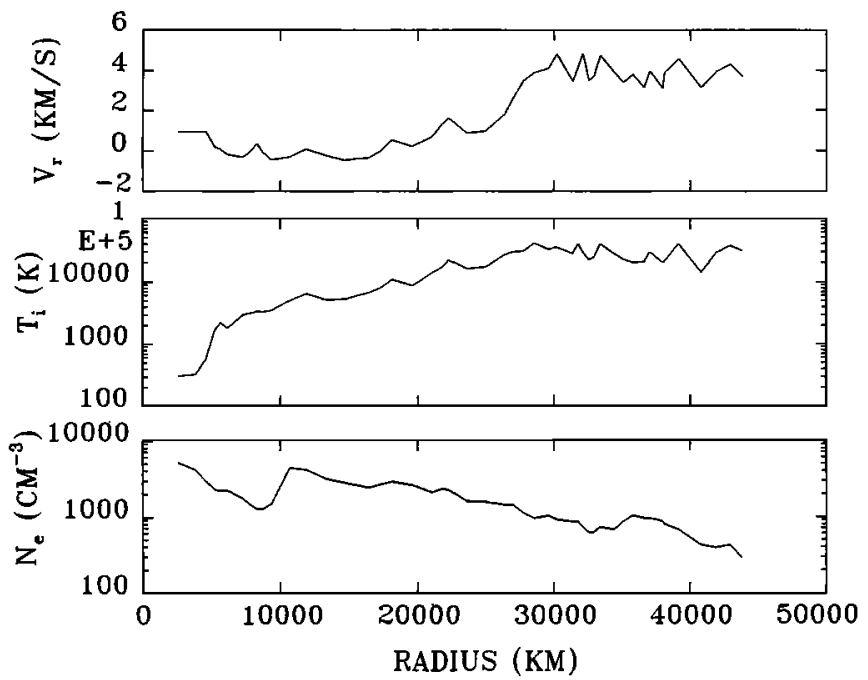

Fig. 1. Data from the high intensity spectrometer of the Giotto ion mass spectrometer (IMS) as a function of cometocentric distance. Adapted from Schwenn et al. (1986). The ion velocity $v_{r}$ is in the spacecraft ram direction (approximately radial). $v_{r}$ and the ion temperature $T_{i}$ are for mass 19 when available (or for mass 17 or 18 otherwise). The relative variation of the electron density, $\mathrm{N}_{e}$, is from the total ion counting rate of the IMS; the absolute scale was set theoretically (Cravens, 1986a).

equation (which contains the magnetic field B) plus the collisional terms appeating in the Körosmezey et al. (1987) energy equation. By using the continuity, momentum (including the B terms), and magnetic field equations, this energy equation can be transformed to one which is in terms of the total time derivative of $T$, and which does not contain $B$ explicitly:

$$
\begin{aligned}
& \frac{3}{2} \mathrm{nk}_{\mathrm{B}}\left\{\frac{\partial \mathrm{T}}{\partial \mathrm{t}}+\overrightarrow{\mathrm{u}} \cdot \nabla \mathrm{T}\right\}+\mathrm{nk}_{\mathrm{B}} \mathrm{T} \nabla \cdot \overrightarrow{\mathrm{u}}+\nabla \cdot \vec{\Phi}=\mathrm{Q}_{\mathrm{ch}}+\mathrm{Q}_{\mathrm{ei}} \\
& +\left(\mathrm{Q}_{\mathrm{in}}-\mathrm{u} \mathrm{F}_{\mathrm{in}}\right)+\left\{\frac{3}{2} \mathrm{k}_{\mathrm{B}}\left(\mathrm{T}_{\mathrm{n}}-\mathrm{T}\right)+\frac{1}{2} \mathrm{~m}\left|\overrightarrow{\mathrm{u}}-\overrightarrow{\mathrm{u}}_{\mathrm{n}}\right|^{2}\right\} S_{\mathrm{c}}(4)
\end{aligned}
$$

where $S_{c}=I_{\text {eff }} n_{n}$ is the ion production rate, $k_{B}$ is Boltzmann's constant, $n$ is the ion/electron number density, and $m, m_{n}$, and $\mu_{\text {in }}$ are the ion, neutral, and reduced masses, respectively. $u$ is the ion velocity and $\Phi$ is the conductive heat flux. $Q_{\mathrm{ei}}$ is the electron-ion energy exchange rate which is not important in the inner coma, and $\left(Q_{i n}-u F_{i n}\right)$ is the ion- neutral collisional energy exchange rate (Banks and Kockarts, 1973) which can be written as:

$$
\left(Q_{i n}-u F_{i n}\right)=\mu_{i n} v_{i n} n\left\{\left(3 k_{B} / m\right)\left(T_{n}-T\right)+\left.\vec{u}_{-u_{n}}\right|^{2}\right\}
$$

$v_{\text {in }}=k_{\text {in }} n_{n}$ is the effective ion-neutral momentum transfer collision frequency with $\mathrm{k}_{\text {in }}=1.05 \times 10^{-9} \mathrm{~cm}^{3} \mathrm{~s}^{-1}$ for $\mathrm{H}_{3} \mathrm{O}^{+}$$\mathrm{H}_{2} \mathrm{O}$ collisions (Cravens and Körösmezey, 1986). The magnetic field does not appear in equation (4) if Ohmic resistive heating is neglected. An estimate of this heating rate near the contact surface, using an expression for the Ohmic resistivity/magnetic diffusion coefficient from Cravens (1986b); gives a value less than $1 \%$ of the chemical heating rate.

\section{Ion Temperature Expression}

The right-hand side of equation (4) is set equal to zero in one version of the local heating approximation. The neglect of the non-local (i.e., dynamical) terms in the ion energy equation should be a reasonable good approximation for the inner coma of comet Halley, except in the immediate vicinity of the contact surface where the gradients of $u$ and $T$ are quite large. The solution of the local heat balance equation (for $m=m_{n}$ ) is:

$$
T-T_{n}=\frac{k_{Q} C_{v}}{3 k_{B} D}+\frac{m_{n}}{3 k_{B}}\left|\vec{u}-\vec{u}_{n}\right|^{2}
$$

where $D=I_{\text {eff }} / 2+\mu_{i n} k_{i n} n / m_{n}$. The first term in this expression represents chemical heating and the second term comes from both frictional heating and heating associated with ion production. The frictional contribution dominates near the CS and the ion production (or mass-loading) contribution is more important at much larger cometocentric distances. The second term on the right-hand side of equation (6) includes, in effect, both frictional and "production" heating associated with relative ion-neutral motions. Haerendel (1987) found a similar expression, and the second term is identical to the "Joule" heating term used in terrestrial ion temperature studies (Banks and Kockarts, 1973; Killeen et al., 1984).

With the values of the parameters discussed earlier, equation (6) for the ion temperature can be rewritten as:

$$
T=T_{n}+\frac{6100 C_{v}}{1+2.1 \times 10^{-3} n}+725\left|\vec{u}-\vec{u}_{n}\right|^{2} K
$$

where the velocities in this equation are in units of $\mathrm{km} / \mathrm{s}$. Now consider the ion temperature given by equation (7) as a function of $u$ in the vicinity of the contact surface of comet Halley (see Figure 2). The ion density is $\mathrm{n}=3.5 \times 10^{3}, \mathrm{~cm}^{-3}$ near the CS (Cravens, 1986a,b) and the neutral temperature $T_{n}$ is in the range $100-300 \mathrm{~K}$ (200 K will be assumed throughout this paper). An initial assumption can be made that both velocities ( $u$ and $u_{n}$ ) were purely radial (positive is outward). The function $T(u)$ has a minimum at $u=u_{n}=0.93 \mathrm{~km} / \mathrm{s}$; that is, where the plasma is flowing outward at the same speed as the neutrals. All the heating is chemical for $\mathfrak{u}=0$, and depends on the value of $\mathrm{C}_{\mathrm{v}}$ assumed. The ion temperatures observed inside the CS ( $r<4000 \mathrm{~km}$ or so -see Figure 1) were $300-400$ $K$ which suggests that $C_{v}$ must be between 0 and 0.5 . $C_{V}=$ 0.2 will be used for the remaining analysis in this paper. The ion velocity drops to zero at the contact surface and Figure 2 suggests that $T$ should jump by $\approx 1000 \mathrm{~K}$ across the CS. The measured T (Balsiger et al, 1986) does increase across the CS, but by $\approx 1500 \mathrm{~K}$ rather than $1000 \mathrm{~K}$. Some of this discrepancy might be due to compressional heating of the ions at the CS,

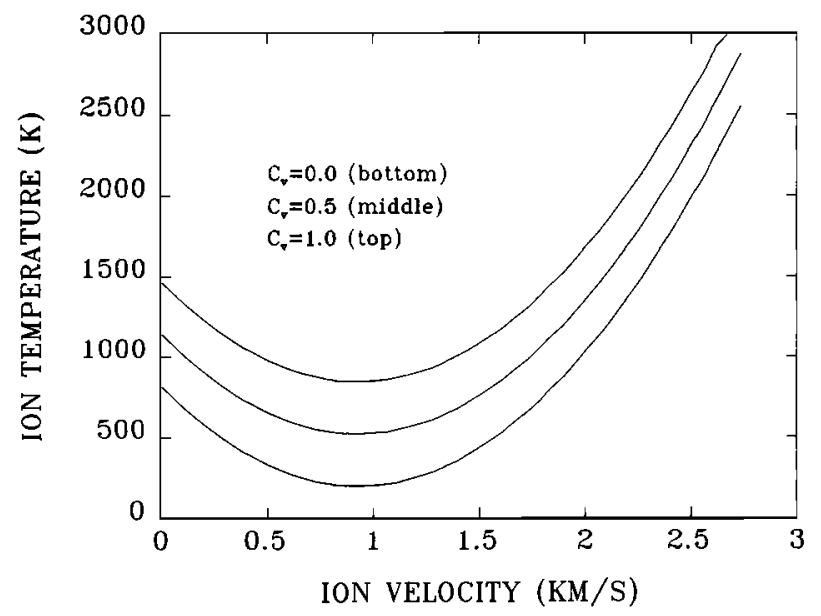

Fig. 2. Theoretical ion temperature versus ion velocity (which is assumed to be purely radial) for three values of the exothermic chemical energy partitioning factor, $C_{v}$. 


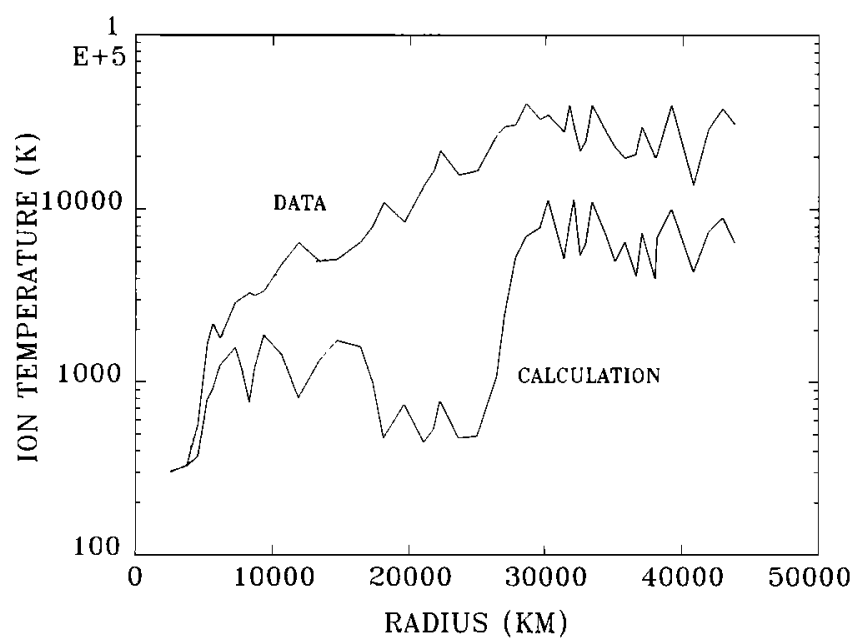

Fig. 3. Theoretical ion temperature as a function of cometocentric distance for $C_{\mathrm{V}}=0.2$ and using $\mathrm{v}_{\mathrm{r}}$ from the Giotto IMS (figure 1). The solid and dashed lines are measured temperature profiles from the Giotto ion (Schwenn et al., 1986) and neutral (Lämmerzahl et al., 1986) mass spectrometers, respectively.

and this might also explain the localized temperature spike observed at the CS by the GIOTTO IMS.

\section{Derived Ion Temperatures}

Equation (7) was used to find $T$ as a function of cometocentric distance (Figure 3 ) using the empirical values of $\mathrm{u}$ and $\mathbf{n}$ from Figure 1 and with $\mathbf{u}$ assumed to be strictly in the radial direction; that is, $\mathrm{u}=\mathrm{v}_{\mathrm{r}}$. The calculated ion temperature sharply increases at the CS as expected and then stays near $1000-2000 \mathrm{~K}$ out to $\mathrm{r} \approx 2.5 \times 10^{4} \mathrm{~km}$, where $\mathrm{T}$ increases to $\approx$ $10^{4} \mathrm{~K}$ due to the increase of $v_{\mathrm{r}}$ to $4 \mathrm{~km} / \mathrm{s}$. The measured temperatures are much larger than the calculated ones for $r$ between $10^{4}$ and $2.5 \times 10^{4} \mathrm{~km}$. This suggests that $u$ is too

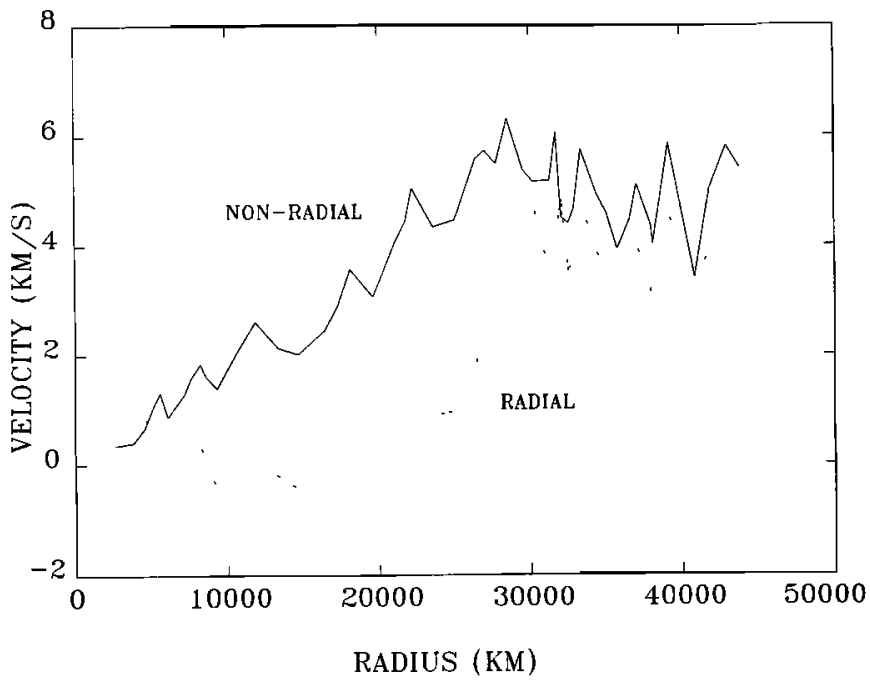

Fig. 4. Transverse plasma velocity profile vs. cometocentric distance, calculated using the theoretical energy balance relation and measured temperatures, densities, and "radial" velocities. The measured radial ion velocity (Schwenn et al., 1986 ) is also shown. small, which further suggests that the plasma velocity must have a significant non- radial component.

The fluctuations in the calculated temperatures are due to the fluctuations in the measured values of $v_{r}$ which were put into equation (7). Notice that in the data (Schwenn et al., 1986; also see Figure 1) positive (outward) excursions in $v_{r}$ are correlated with positive excursions in $\mathrm{T}$. However, if the plasma moved only in the radial direction, equation (7) predicts that for $v_{r} \approx 0$, small positive excursions of $v_{r}$ must be associated with decreases in $T$. This again supports the existence of a non-radial velocity component which is usually positively correlated with $v_{\mathbf{r}}$.

\section{Discussion}

One possible reason for the discrepancy between the measured and calculated ion temperatures in Figure 3 is the existence of plasma motions which are transverse, or orthogonal, to the Giotto trajectory (which is approximately radially-directed). The velocity vector can be written :

$$
\overrightarrow{\mathbf{u}}=\mathbf{v}_{\mathbf{r}} \hat{\mathbf{r}}+\mathbf{v}_{\mathbf{T}} \hat{\mathbf{n}}
$$

$\hat{\mathbf{r}}$ and $\hat{\mathrm{n}}$ are unit vectors radially and orthogonally-directed.

The empirical values of $T, n$, and $v_{r}$ (from Figure 1) were substituted into equation (7), which was then solved for $\mathrm{vT}_{\mathrm{T}}$. The resulting derived non-radial (horizontal) velocities are shown in Figure 4. There are many sources of error in these results, other than instrumental error, such as the neglect of non-local terms in the energy balance equation, the neglect of high-speed correction terms in the collision frequency, the neglect of electron-ion energy exchange, possible waveparticle heating, etc., although estimates indicate that these errors should be rather small.

Haerendel (1987) also explained the ion temperatures in the inner coma with ion velocities of about $3 \mathrm{~km} / \mathrm{s}$, but the plasma motion in that study was postulated to be radially inward. His dynamical analysis pertained to whole flux tubes, which are "draped" around the inner coma, and the plasma motion along the sun-comet axis must indeed be radial. But in the flanks of the magnetic barrier (which is where the Giotto spacecraft traversed the inner coma) plasma flow primarily in the antisunward (i.e., non-radial) direction seems more likely.

The momentum equation can be written, very approximately, in the form of a diffusion equation if the mass-loading terms (as considered by Haerendel, 1986, for instance) are ignored. The transverse velocity is then proportional to the transverse (non-radial) component of the force on the plasma:

$$
\vec{v}_{T}=\frac{1}{n m v_{\text {in }}}\{-\nabla p+\vec{J} \times \vec{B}\}_{T}
$$

where the current density $\overrightarrow{\mathrm{J}}=\nabla \times \overrightarrow{\mathrm{B}} / \mu_{0}$.

The J x B force includes both the magnetic pressure gradient and magnetic curvature forces. A rough estimate of vT using equation (8) indicates that it should be less than $\approx 1 \mathrm{~km} / \mathrm{s}$ near the contact surface, where the $J \times$ X force is predominantly radial and where the collision frequency $\left(v_{\text {in }}\right)$ is large. But, at larger cometocentric distances, $v_{\text {in }}$ becomes much smaller and the forces in the non-radial direction larger and values of $v_{T}$ of $\approx \mathrm{few} \mathrm{km} / \mathrm{s}$ are reasonable.

Acknowledgments. This work was supported by NASA grants NAGW-15, NGR 23-005-015, and NSF grant ATM 8417884. I would like to thank Phyllis Cory for editorial assistance with the manuscript and Marcus Kurtz for computational assistance. 


\section{REFERENCES}

Balsiger, H., K. Altwegg, F. Bühler, J. Geiss, A.G. Ghielmetti, B.E. Goldstein, R. Goldstein, W.T. Huntress, W.-H. Ip, A.J. Lazarus, A. Meier, M. Neugebauer, U. Rettenmudd, H. Rosenbauer, R. Schwenn, R.D. Sharp, E.G. Shelley, E. Ungstrup, and D.T Young, Ion composition and dynamics at comet Halley, Nature, 321, 330, 1986.

Banks, P.M., and G. Kockarts, Aeronomy, Academic Press, New York, 1973.

Cravens, T.E. and A. Körösmezey, Vibrational and rotational cooling of electrons by water vapor, Planet. Space Sci., 34 961, 1986.

Cravens, T.E., Theory and observation of cometary ionospheres, XXVI COSPAR Meeting, Toulouse, France, 1986a.

Cravens, T.E., The physics of the cometary contact surface, Proc. 20th ESLAB Symposium on the exploration of Halley's comet, ESA SP-250, 241, 1986b.

Cravens, T.E., J.U. Kozyra, A.F. Nagy, T.I. Gombosi, and M. Kurtz, Electron Impact ionization in the vicinity of comets, J. Geophys. Res., 92, 7341, 1987.

Gringauz, K.I., T.I. Gombosi, A.P. Remizov, I. Apáthy, I Szemerey, M.I. Verigin, L.I. Denchikova, A.V. Dyachkov, E. Keppler, I.N. Klimenko, A.K. Richter, A.J. Somogyi, K. Szegö, S. Szendrö, M. Tátrallyay, A. Varga, and G.A. Vladimirova, First in situ plasma and neutral gas measurements at comet Halley, Nature, 321, 282, 1986.

Haerendel, G., Plasma flow and critical velocity ionization in cometary comae, Geophys. Res, Lett., 13, 255, 1986.

Haerendel, G., Plasma transport near the magnetic cavity surrounding comet Halley, Geophys. Res, Lett., 14, 673, 1987.

Ip, W.-H., and W.I. Axford, The formation of a magnetic field free cavity at comet Halley, Nature, 325, 418, 1987.

Killeen, T.L., P.B. Hays, G.R. Carignan, R.A. Heelis, W.B. Hanson, N.W. Spencer, and L.H. Brace, Ion-neutral coupling in the high-latitude $\mathrm{F}$ region: Evaluation of ion heating terms from Dynamics Explorer 2, J. Geophys. Res., 89, 7495, 1984.

Körösmezey, A., T.E. Cravens, T.I. Gombosi, A.F. Nagy, D.A. Mendis, K. Szegö, B.E. Gribov, R.Z. Sagdeev, V.D. Shapiro, and V.I. Shevchenko, A comprehensive model of cometary ionospheres, J. Geophys. Res., 92,

Krankowsky, D., P. Lämmerzahl, L. Herrwerth, J. Woweries, P. Eberhardt, U. Dolder, U. Hermann, W. Schulte, J.J. Berthelier, J.M. Illiano, R.R. Hodges, and J.H. Hoffman, In situ gas and ion measurements at comet Halley, Nature, 321, 326, 1986.

Lämmerzahl, P. et al., Expansion velocity and temperatures of gas and ions measured in the coma of comet Halley, Proc. 20th ESLAB Symposium on the exploration of Halley's comet, ESA SP-250, 179, 1986.

Mendis, D.A., H.L.F. Houpis, and M.L. Marconi, The physics of comets, Fund. Cosmic Phys, 10, 1, 1985.

Neubauer, F.M., K.H. Glassmeier, M. Pohl, J. Raeder, M.H. Acuna, L.F. Burlaga, N.F. Ness, G. Musmann, F. Mariani, M.K. Wallis, E. Ungstrup, and H.U. Schmidt, First results from the Giotto magnetometer experiment at comet Halley, Nature, 321, 352, 1986.

Schmidt, H.U., and R. Wegmann, Plasma flow and magnetic fields in comets, in Comets, ed. L.L. Wilkening, pp 538560, University of Arizona Press, Tucson, 1982

Schwenn, R., W.-H. Ip, H. Rosenbauer, H. Balsiger, F. Bühler, R. Goldstein, A. Meier, and E.G. Shelley, Ion temperature and flow profiles in comet Halley's close environment, Proc. 20th ESLAB Symposium on the exploration of Halley's comet, ESA SP-250, 225, 1986.

T.E. Cravens, The University of Michigan, Department of Atmospheric and Oceanic Science, Space Physics Research Laboratory, Ann Arbor, Michigan 48109-2143.

(Received May 27, 1987; revised July 16,1987 ; accepted August 3,1987.) 